\title{
Diferencias individuales en modelos animales: un enfoque para el estudio de factores neurobiológicos relacionados con depresión
}

\author{
Individual differences in animal models: an approach to \\ study neurobiological factors related to depression
}

\author{
Andrey Sequeira Cordero ${ }^{1}$ \\ Jaime Fornaguera Trías ${ }^{2}$ \\ Universidad de Costa Rica, Costa Rica
}

\begin{abstract}
Resumen. El estudio de las diferencias individuales en la prueba de nado forzado (PNF) en ratas permite la identificación de factores de susceptibilidad o de resistencia en el desarrollo conductas relacionadas con la depresión. Para estudiar estas diferencias, varios grupos de ratas fueron sometidos a la PNF; posteriormente, los animales con baja y alta inmovilidad fueron comparados, lo que permitió identificar una serie de características que podrían estar actuando como factores de riesgo o de protección. Así, factores neurobiológicos como la expresión diferencial en el núcleo accumbens del receptor 1 para el factor liberador de corticotropina (CRFR1), una tasa diferencial de recambio de dopamina en esa misma región y la cinética de expresión diferencial del factor neurotrófico derivado del cerebro (BDNF) en la corteza prefrontal, podrían jugar un papel importante en la modulación de conductas depresivas. La presente revisión resume nuestros resultados más importantes en esta línea de investigación.
\end{abstract}

Palabras clave. Diferencias individuales, prueba de nado forzado, conducta, expresión génica, cerebro.

Abstract. The study of individual differences in the forced swimming test (FST) in rats allows the identification of either susceptibility or resilience factors in the development of depression-related behaviors. In order to study these differences several rat groups were subjected to the FST. Afterward, animals with low and high immobility were compared, which allowed us to identify a number of features that could function as risk or protection factors. Thus, neurobiological factors such as the expression levels of the corticotropin-releasing factor receptor 1 (CRFR1) in the nucleus accumbens, a differential accumbal dopamine turnover and the differential expression kinetics of the brain-derived neurotrophic factor (BDNF) in the prefrontal cortex, could play an important role in the modulation of depressive behaviors. The current review summarizes our key results in such research line.

Keywords. Individual differences, forced swimming test, behavior, gene expression, brain.

\footnotetext{
${ }^{1}$ Andrey Sequeira Cordero, Instituto de Investigaciones en Salud (INISA), Centro de Investigación en Neurociencias (CIN), Universidad de Costa Rica. Direccion postal: 11501-2060, San Pedro, San José, Costa Rica. E-mail: bioaseq@gmail.com

${ }^{2}$ Jaime Fornaguera Trías, Instituto de Investigaciones en Salud (INISA), Centro de Investigación en Neurociencias (CIN), Escuela de Medicina, Universidad de Costa Rica. E-mail: jaime.fornaguera@ucr.ac.cr
} 


\section{Introducción}

La depresión es una enfermedad muy común que afecta entre el 15 y el $20 \%$ de la población general (Andrade et al., 2003; Kessler et al., 2003), aunque dicha prevalencia varía en diferentes poblaciones (Lépine \& Briley, 2011). En Costa Rica el panorama es similar, de hecho, según datos del Consejo Nacional de la Niñez y la Adolescencia (2009), esta enfermedad representa un problema importante en la población joven, en la cual se ha observado una prevalencia de hasta el $20 \%$ y un incremento de hasta un $45 \%$ de jóvenes que requieren atención psicológica. La alta frecuencia de la depresión y el efecto significativo en la calidad de vida de las personas (Lépine \& Briley, 2011), ponen en evidencia la necesidad de realizar investigaciones, tanto a nivel clínico como también preclínico, con el objetivo de profundizar en el conocimiento sobre los mecanismos y factores relacionados con el desarrollo de la enfermedad y al mismo tiempo, mejorar los tratamientos ya existentes o diseñar nuevas herramientas terapéuticas. Desde esta perspectiva, el enfoque de las diferencias individuales se perfila como un abordaje de gran importancia en la realización de dichas investigaciones.

El estudio de las diferencias individuales utiliza la variabilidad observada en una característica fenotípica (i.e., endofenotipo) de interés, para agrupar a los individuos de una cohorte según el grado o nivel en que presenten dicha característica (e.g., altos versus bajos) de forma que al comparar ambos grupos, se pueden identificar factores relacionados con esa diferenciación (revisado en Pawlak, Ho \& Schwarting, 2008; Harro, 2010). Lo anterior adquiere importancia cuando el endofenotipo escogido se relaciona con una condición o con una patología cuya base biológica se quiere dilucidar, además de que también permite identificar factores de susceptibilidad o de resistencia a ella (Shishkina, Kalinina, Berezova, Bulygina \& Dygalo, 2010). Aunque este enfoque puede ser utilizado en estudios con humanos, buena parte del trabajo realizado hasta hoy se ha llevado a cabo usando modelos animales; estos modelos han sido desarrollados principalmente para el estudio de la ansiedad y en menor grado, de la depresión (revisado en Armario \& Nadal, 2013). Así por ejemplo, a través del estudio de las diferencias individuales en la prueba del laberinto elevado en cruz se logró corroborar la relación entre conductas asociadas con la ansiedad y los niveles de serotonina (5-HT) en el estriado ventral, en donde animales que permanecen menos tiempo en los brazos abiertos (es decir, animales más ansiosos), presentaron menores niveles de 5-HT en el estriado ventral en comparación con animales menos ansiosos (Schwarting et al., 1998).

Del mismo modo, el estudio de las diferencias individuales en la prueba de la desesperanza aprendida (niveles de inmovilidad incrementada en respuesta a choques eléctricos en las patas) ha permitido determinar que animales con altos niveles de desesperanza presentan un menor consumo de sacarosa, lo cual se utiliza como medida de la anhedonia (incapacidad para experimentar placer), lo que sugiere que su uso en el estudio de factores relacionados con esta conducta asociada con la depresión es adecuado (Shumake, Barrett, \& Gonzalez-Lima, 2005). En este modelo, animales susceptibles a desarrollar la desesperanza presentan una menor actividad de la enzima citocromo oxidasa en varias regiones corticales (Shumake, Poremba, Edwards, \& Gonzalez-Lima, 2000), así como un mayor metabolismo oxidativo en hipocampo y en subículum en comparación con ratas resistentes a la desesperanza (Shumake, Edwards, \& Gonzalez-Lima, 2002). De esta manera, los ejemplos anteriores evidencian que el enfoque de las diferencias individuales permite identificar factores neuroconductuales relacionados con fenotipos de interés en el modelaje de psicopatologías como la ansiedad y la depresión.

Además, es importante mencionar que otros grupos se han centrado en distintos endofenotipos relacionados con otros procesos de importancia para el ser humano, como lo son la sensibilidad a sustancias psicoestimulantes (Mandt, Allen \& Zahniser, 2009) y el aprendizaje y la memoria (Fattore, Piras, Corda \& Giorgi, 2009), entre otros. En lector interesado en profundizar en la utilización del enfoque de las diferencias individuales en diferentes modelos puede remitirse a excelentes revisiones publicadas recientemente (Pawlak, Ho \& Schwarting, 2008; Harro, 2010; Armario \& Nadal, 2013). 
Desde la perspectiva anterior, nuestro grupo ha venido estudiando las diferencias individuales en ratas macho en conductas relacionadas con la depresión (la realización de este tipo de estudios en hembras se complica debido el efecto del ciclo estral en las respuestas neuroconductuales al estrés, de ahí que nos hayamos enfocado en el estudio de machos solamente). A continuación se resumen los resultados más importantes y las implicaciones de los mismos con respecto a este padecimiento.

Diferencias individuales en conductas relacionadas con la depresión: la PNF

La prueba de nado forzado (PNF) desarrollada por Porsolt, Le Pichon \& Jalfre, (1977) consiste en introducir a un animal (generalmente un roedor) en un recipiente con agua del que no puede escapar. El animal lucha por hacerlo, presentando una serie de conductas características como el nado, intentos de escalamiento por las paredes del recipiente, e incluso el buceo, alternando con periodos en los que se queda inmóvil. En una segunda sesión, realizada veinticuatro horas después (conocida como la prueba, pues la primera se denomina preprueba), la inmovilidad es considerada una conducta de desesperanza (i.e., desesperanza aprendida) y está modulada por factores y mecanismos relacionados con la depresión (Rupniak, 2003) (Figura 1A).

Esta interpretación ha sido ampliamente validada a través de estudios farmacológicos, en donde drogas con actividad antidepresiva en humanos, disminuyen significativamente el tiempo de inmovilidad en la prueba (Slattery \& Cryan, 2012). En este sentido, es importante aclarar que la PNF no es un modelo de depresión propiamente dicho, aunque sí permite el estudio de factores relacionados con esta enfermedad.

Como se mencionó anteriormente, se han realizado algunas investigaciones sobre diferencias individuales usando como endofenotipo de interés la inmovilidad en la PNF (Weiss, Cierpial, \& West, 1998; Taghzouti, Lamarque, Kharouby \& Simon, 1999; Naudon \& Jay 2005; Shishkina et al., 2010). De esta manera, mediante la comparación de animales con baja inmovilidad (i.e., animales que presentan niveles reducidos de la conducta relacionada con la depresión) con animales con alta inmovilidad (i.e., animales con mayores niveles de la conducta relacionada con la depresión) (Fig. 1), se observaron resultados interesantes.

Por ejemplo, Taghzouti y colaboradores (1999) describen que animales con alta o baja inmovilidad responden de manera diferencial a la fluoxetina (uno de los fármacos más ampliamente utilizados en humanos para el tratamiento de la depresión), lo cual evidencia mecanismos cerebrales distintos en cuanto a la respuesta al estrés y/o el despliegue de conductas relacionadas con la depresión. Por su parte, Naudon y Jay (2005) hallaron que ratas con alta inmovilidad también presentan mayores dificultades para aprender tareas relacionadas con memoria espacial y memoria de trabajo, lo cual señala que el modelo podría utilizarse incluso para estudiar la relación depresión-memoria tan ampliamente descrita (para un meta-análisis ver Rock, Roiser, Riedel \& Blackwell, 2013).

Desde una perspectiva molecular, Shishkina et al. (2010) lograron identificar diferencias en cuanto a los niveles de proteínas específicas relacionadas con el efecto del estrés agudo a nivel hipocampal. Los resultados anteriores permiten caracterizar las diferencias entre animales con alta y baja inmovilidad lo que en última instancia, permitiría no sólo entender mejor los mecanismos relacionados con la depresión sino al mismo tiempo, empezar a comprender por qué algunos individuos son más propensos que otros a desarrollar conductas depresivas como consecuencia del estrés. Tal conocimiento representaría un avance sin precedentes en el estudio de las bases neurobiológicas de la depresión.

Recientemente, hemos utilizado las diferencias individuales en la PNF con el objetivo de colaborar con la caracterización conductual y neuroquímica de ratas macho con baja y alta inmovilidad. A través de una serie de experimentos, logramos identificar blancos moleculares no descritos hasta ahora en este contexto, lo cual nos ha permitido proponer una serie de hipótesis con respecto a los posibles mecanismos asociados con el desarrollo de conductas relacionadas con la depresión. 


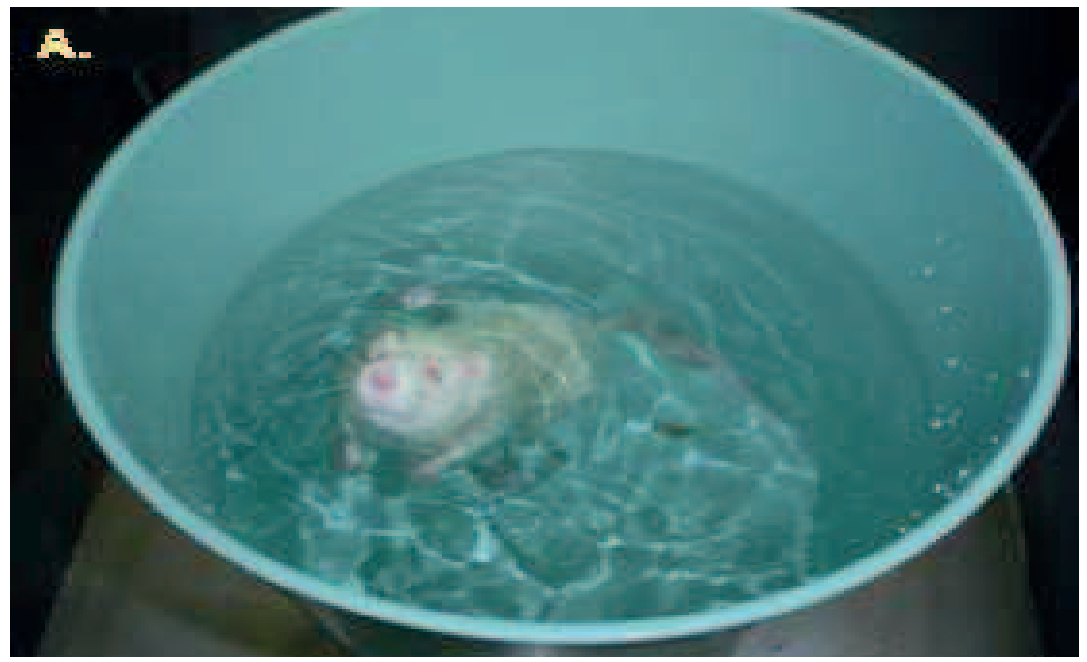

B.

Inmovilidad PNF

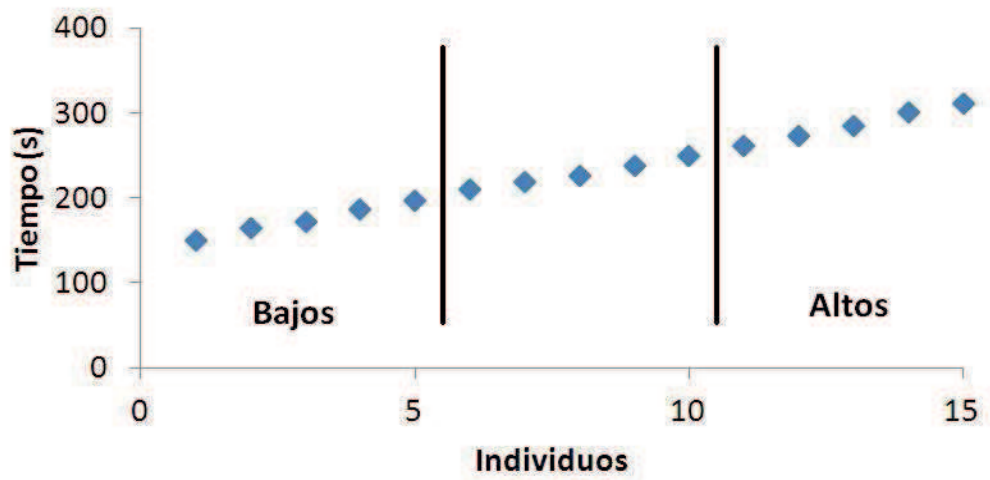

Figura 1. Diferencias individuales en la prueba de nado forzado. A) En la conducta de inmovilidad el animal solo realiza los movimientos necesarios para mantenerse a flote. B) Los animales se agrupan según el tiempo de inmovilidad en la PNF en baja y alta inmovilidad, respectivamente (para una revisión detallada sobre los criterios de clasificación ver Harro, 2010).

Diferencias entre individuos con alta y baja inmovilidad en la PNF

Uno de los objetivos más frecuentes al estudiar las diferencias individuales consiste en determinar si las diferencias en la conducta de clasificación, en nuestro caso la inmovilidad en la PNF, reflejan además diferencias en otras conductas medidas en la misma prueba, o en pruebas relacionadas con otros endofenotipos, como la ansiedad o la memoria. El hecho de que la clasificación en cuanto a inmovilidad permita identificar diferencias en otras conductas, sugiere que éstas comparten una base neurobiológica, es decir, que los factores mediadores son hasta cierto punto, compartidos; lo que podría facilitar la identificación de elementos relacionados con la comorbilidad de dos patologías, como la depresión y la ansiedad.

En este sentido, una observación que se repite en nuestros estudios consistentemente, es la de que 
las diferencias individuales en la PNF no predicen diferencias en la prueba de campo abierto (PCA) (Sequeira-Cordero, Mora-Gallegos, Cuenca-Berger \& Fornaguera-Trías, 2013; 2014a; datos no publicados). La PCA es un paradigma que permite medir la actividad y algunas conductas de exploración cuya interpretación da información sobre los niveles de ansiedad que manifiesta el animal (Walsh \& Cummins, 1976).

A primera vista, estos resultados indican que conductas relacionadas con la depresión y aquellas asociadas con la ansiedad, no comparten una base neurobiológica, al menos en lo que respecta a lo que estas pruebas pueden medir; lo cual no concuerda con el hecho de que en humanos la depresión y el síndrome de ansiedad presentan una alta comorbilidad (Hirschfeld, 2001). De manera interesante, en cada experimento que realizamos la PCA se llevó a cabo dos días antes de la PNF para evitar un efecto de arrastre (la PNF implica altos niveles de estrés por lo que sus efectos podrían alterar el resultado de pruebas realizadas dentro de cierto periodo posterior). Sin embargo, en un experimento independiente en el cual la PCA se llevó a cabo una semana después sí observamos diferencias: animales con baja inmovilidad presentaron mayores niveles de conductas que se consideran indicadores de ansiedad como mayores niveles de locomoción (Sequeira-Cordero, MoraGallegos, Cuenca-Berger \& Fornaguera-Trías, 2014b). Dicha observación inesperada apunta que el efecto que la PNF en sí produce en los sujetos podría mantenerse hasta al menos una semana después de la misma y, más importante todavía, que dicho efecto podría potenciar de alguna manera las diferencias entre animales con baja y alta inmovilidad en su respuesta al campo abierto. Lo anterior sugiere una base neurobiológica parcialmente común (i.e., al ser respuestas conductuales diferentes a estímulos distintos, también involucran factores específicos no compartidos) que, para hacerse evidente a nivel conductual, debe ser potenciada por un estímulo estresante previo. Esto tiene sentido si se toma en cuenta que según R. Schwartig (comunicación personal, 2013) en humanos las conductas depresivas y las ansiosas tienen una alta comorbilidad solamente en el estado patológico.
A la hora de identificar los elementos neurobiológicos relacionados con las diferencias individuales en la PNF, es importante tomar en cuenta la gran cantidad de factores involucrados en el desarrollo de la depresión (Sequeira \& Fornaguera, 2009). En ese sentido hay que mencionar que las diferentes investigaciones deben enfocarse a distintos niveles, desde regiones cerebrales y circuitos neurológicos como el hipocampo y la amígdala en el sistema límbico, hasta moléculas específicas como neuropétidos, neurotrasmisores, hormonas y/o microARNs, entre otros, que operan a nivel de poblaciones celulares específicas (Sequeira \& Fornaguera, 2009; Villanueva, 2013). Desde esta perspectiva, nuestros estudios se centraron en tres áreas del cerebro: el hipocampo, la corteza prefrontal y el núcleo accumbens. Estas regiones han sido consistentemente identificadas como parte del circuito que tiene una función fundamental en el control de la conducta y el desarrollo de psicopatologías como la depresión y el síndrome de ansiedad (Bennett, 2011).

Por otro lado, nos enfocamos en el factor liberador de corticotropina (CRF, por sus siglas en inglés) y su receptor 1 (CRFR1), el factor neurotrófico derivado del cerebro (BDNF) y su receptor tirosínkinasa relacionado con tropomiosina ( $\operatorname{TrkB}$ ) y el contenido de neurotransmisores monoaminérgicos, i.e., dopamina (DA), norepinefrina (NE) y 5-HT.

Animales con baja inmovilidad presentaron niveles significativamente mayores del ARNm para el CRFR1 en el núcleo accumbens, lo cual indica que la expresión de este gen está incrementada en animales "menos deprimidos" (Sequeira-Cordero et al., 2013). El CRFR1 es un receptor acoplado a proteínas G que, junto con otro receptor conocido como el CRFR2, regula la acción del CRF en diferentes regiones del cerebro (Aguilera, Nikodemova, Wynn \& Catt, 2004). La unión del péptido al CRFR1 activa distintas vías de señalización que a su vez, regulan una amplia variedad de procesos fisiológicos y conductuales relacionados con la respuesta al estrés y las conductas depresivas (Grammatopoulos \& Chrousos, 2002). Las funciones del sistema CRF-CRFR1 dependen del sitio específico del cerebro en el cual se dé la activación o la inactivación del mismo (Aguilera et al., 2004). El 
núcleo accumbens tiene una importante función en los procesos relacionados con la recompensa, aunque también participa en la regulación de la respuesta al estrés (Muschamp et al., 2011).

Una posible interpretación de nuestros datos sería que la baja en la expresión del CRFR1 (y por lo tanto en las vías de señalización desencadenadas por CRF) en el núcleo accumbens, se asocia con un incremento en los niveles de desesperanza aprendida, es decir, la reducción en los niveles de CRFR1 participaría en el despliegue de conductas relacionadas con la depresión. Sin embargo, la función de CRF en el núcleo accumbens ha sido recientemente asociada con la motivación positiva dirigida a enfrentar eventos estresantes (Peciña, Schulkin \& Berridge, 2006; Lemos et al., 2012).

Estas observaciones permiten complementar la interpretación anterior pues sugieren que el incremento en la expresión de CRFR1 en esta región y el esperable incremento en la señalización, podrían mediar la motivación en busca de recompensa que en el caso de la PNF, estaría representada por el escape de la situación estresante. Consecuentemente, esto lleva a que el animal manifieste conductas de enfrentamiento más activas (i.e., baja inmovilidad junto con mayores niveles de nado y/o escalamiento). Por el contrario, animales con bajos niveles de expresión de CRFR1 en el núcleo accumbens presentarían menor motivación a la hora de buscar la recompensa, lo cual se traduce en mayores tiempos de inmovilidad. En estos procesos de control neuroconductual también está involucrada la neurotrasmisión de dopamina, aunque de manera compleja, pues su participación depende de factores como el desarrollo y la severidad del estímulo estresante (Lemos et al., 2012; Sequeira-Cordero et al., 2013).

El papel del sistema CRF-CRFR1 no solamente es importante en relación con las diferencias individuales sino que además, podría tener al mismo tiempo una función mediadora de los efectos del enriquecimiento ambiental (Sequeira-Cordero et al., 2014b). El ambiente es capaz de modificar la expresión de los genes y esta interacción genes-ambiente es la que determina la mayoría (sino todos) de los rasgos fenotípicos de los individuos (Zhang \& Meaney, 2010).
El enriquecimiento ambiental, es decir, la exposición de los individuos a estímulos físicos y sociales a los que no están expuestos normalmente, ha demostrado tener un importante efecto en el funcionamiento cerebral (Simpson \& Kelly, 2011). En este contexto, nuestro grupo estudió también el efecto del enriquecimiento ambiental en animales con baja y alta inmovilidad en la PNF con el objetivo de buscar una interacción entre las diferencias individuales y la respuesta al alojamiento, lo cual evidenciaría una interacción que biológicamente, se traduce como la presencia de factores que no sólo son importantes como moduladores de la conducta (alta o baja inmovilidad), sino también como mediadores del efecto ambiental. Nuestros resultados mostraron que animales con baja inmovilidad enriquecidos tienen menores niveles de CRF en el núcleo accumbens si se comparan con animales alojados en condiciones estándar (i.e., alojados en grupos de cuatro y sin acceso a los estímulos propios de la caja de enriquecimiento), mientras que animales con alta inmovilidad enriquecidos presentaron una tendencia marginalmente significativa al incremento de CRFR1 en el núcleo accumbens comparados con sus contrapartes en condiciones estándar (Sequeira-Cordero et al., 2014b).

En términos generales, los datos sugieren que animales con baja inmovilidad alojados en condiciones estándar presentan una respuesta moderada del sistema CRF-CRFR1 (tanto en lo referente a la síntesis del receptor en el núcleo accumbens, como también a la síntesis del péptido señal que puede actuar en otras regiones del cerebro). Por otro lado, animales con alta inmovilidad que fueron enriquecidos presentan una respuesta más exacerbada. Estas observaciones señalan a la señalización activada por CRF-CRFR1 como una vía importante en el despliegue de la conducta de inmovilidad y en la mediación del efecto del ambiente enriquecido.

Adicionalmente, a la hora de identificar factores relacionados con las diferencias individuales, el estudio de la cinética neuroquímica (i.e., el cambio a lo largo del tiempo de diferentes factores neuroquímicos luego de la exposición a un estímulo específico) debe ser tomado en cuenta. Este tipo de abordajes tiene como objetivo 
determinar si únicamente las concentraciones de una o de varias sustancias, o si también el comportamiento en un periodo de tiempo definido, permiten distinguir entre las categorías de baja y alta inmovilidad.

Para identificar este tipo de variaciones estudiamos la cinética de los diferentes factores neuroquímicos en tres momentos: una hora, seis horas y veinticuatro horas después de la PNF. Además, para realizar una comparación con animales no sometidos al estrés del nado forzado se utilizó un grupo control que no fue sometido a la prueba. Nuestras observaciones indican una diferencia en la cinética de expresión del BDNF en la corteza prefrontal y en el núcleo accumbens (Sequeira-Cordero et al., 2014a). El BDNF es un péptido que al unirse a su receptor $\operatorname{TrkB}$ regula una serie de procesos relacionados con la plasticidad, la sobrevivencia neuronal y la sinaptogénesis, entre otros. Tiene un importante impacto en la conducta y juega un papel relevante en el desarrollo de psicopatologías como la depresión (revisado en Lee \& Kim, 2010).

En nuestros estudios se observó una disminución en los niveles de BDNF en la corteza prefrontal de los animales sometidos al estrés por nado. La reducción significativa observada apareció una hora después de la PNF en animales con baja inmovilidad, mientras que a las 6 horas dicha reducción se observó, tanto en los animales de baja inmovilidad, como también en los de alta inmovilidad. Finalmente a las veinticuatro horas, los niveles de ARNm de BDNF en ambos grupos vuelven a ser similares a los del grupo control no sometido a la prueba. Por otro lado, en el núcleo accumbens solamente los animales con baja inmovilidad presentaron una disminución de los niveles de BDNF seis horas después de haber sido sometidos a la PNF.

El despliegue de la inmovilidad en la PNF puede considerarse como un marcador conductual de la activación de varios mecanismos relacionados con la aparición de conductas depresivas. Nuestros datos sugieren una dinámica distinta entre animales con alta y baja inmovilidad donde el efecto relacionado con la PNF (i.e., la reducción en la neurotrofina) se da con una cinética temporal distinta. Es importante mencionar una discrepancia con respecto a lo esperado; la disminución en los niveles de BDNF ha sido ampliamente asociada con el surgimiento de la depresión (Yu \& Chen, 2011) y desde ese punto de vista, es de esperar que los animales "más deprimidos" experimenten una disminución mayor o al menos más rápida de esta neurotrofina.

En nuestro caso, observamos justamente lo contrario: los "menos deprimidos" (animales con baja inmovilidad) presentaron la reducción más tempranamente. Nuevos experimentos deben ser diseñados con el objetivo de recabar más información para que estas observaciones puedan ser interpretadas de manera más precisa.

\section{La edad como modulador fundamental}

La edad representa un importante factor mediador de cambios en el funcionamiento neuroconductual (Casey, Jones \& Hare, 2008), por lo tanto, la respuesta ante un estímulo de cualquier tipo puede variar a lo largo de las diferentes etapas del desarrollo. Esto es particularmente cierto en los primeros estadios como el desarrollo fetal, la infancia y la adolescencia, en los cuales los individuos presentan una alta vulnerabilidad al efecto de factores ambientales estresantes, (Casey et al., 2008; Dipietro, 2012).

Basados en esta premisa, nuestro grupo realizó varios experimentos para determinar si los factores relacionados con las diferencias individuales se mantienen entre individuos adolescentes y adultos jóvenes. De acuerdo con el desarrollo de las ratas, las edades a las cuales los animales fueron sometidos a la PNF, es decir los días posnatales 32 y 92, representan las etapas prepuberal y de adulto joven, respectivamente (Schneider, 2013). Desde la perspectiva conductual, animales jóvenes presentaron tiempos de inmovilidad en la prueba significativamente mayores que los individuos adultos (Sequeira-Cordero et al., 2013); lo que sugiere que la adolescencia es un periodo del desarrollo en el que los individuos son más propensos a presentar conductas depresivas ante la exposición al estrés, lo cual también ha sido postulado para seres humanos (Sun \& Alkon, 2008; Jankord et al., 2011).

La mayor vulnerabilidad de los individuos podría estar asociada con el hecho de que varias regiones corticales 
y subcorticales (e.g., el sistema límbico) aún no han alcanzado su madurez durante la adolescencia, y sólo la completan hasta que se alcanza el estado adulto (Casey et al., 2008). Esta inmadurez podría explicar, al menos parcialmente, las diferencias entre ambos grupos etarios y la mayor vulnerabilidad en los adolescentes.

Interesantemente, las diferencias entre grupos etarios a nivel conductual (no solamente con respecto a la inmovilidad sino también a las otras conductas observadas en la PNF y a todas aquellas medidas en la PCA) se vieron reflejadas en las profundas diferencias a nivel neuroquímico halladas al comparar los dos grupos etarios, en donde los neurotransmisores estudiados (serotonina, dopamina y norepinefrina) presentaron niveles significativamente diferentes entre adolescentes y adultos (las diferencias dependen del neurotransmisor y de la región, algunos presentaron incrementos y otros disminuciones) (Sequeira-Cordero et al., 2013).

Lo anterior sugiere que las diferencias conductuales podrían estar relacionadas al menos en una parte, con diferencias en la neurotransmisión monoaminérgica asociadas con la edad; esto ha sido ha sido propuesto también por otros autores (Topic, Oitzl, Meijer, Huston \& de Souza Silva, 2008; Arrant, Jemal \& Kuhn, 2013). La comparación entre altos y bajos también arrojó datos interesantes al tomar en cuenta el factor edad. Es bien sabido que la conducta en la PNF puede cambiar marcadamente de la preprueba a la prueba, en donde las conductas pasivas (inmovilidad) se incrementan sustancialmente, en tanto que las activas (escalamiento, nado, buceo) se reducen (Porsolt et al., 1978). Lo que como ya se mencionó antes, está relacionado con la naturaleza inescapable de la prueba y la adquisición de la desesperanza (Armario \& Nadal, 2013).

Esta tendencia al incremento de la inmovilidad y la reducción de conductas activas se observó en animales adolescentes y en adultos con alta inmovilidad. Sorpresivamente, ratas adultas con baja inmovilidad no presentaron ningún cambio conductual entre preprueba y prueba (Sequeira-Cordero et al., 2013). Esta inesperada observación parece apoyar la idea de que en adultos jóvenes los mecanismos que controlan las estrategias de enfrentamiento activo a situaciones estresantes repetidas (i.e., dos sesiones del nado forzado) podrían funcionar como factores protectores que reducen la probabilidad de desarrollar conductas relacionadas con la depresión. Dicho de otra forma, un adulto joven que enfrenta una situación de estrés de manera más activa, tiene menor probabilidad de desarrollar una conducta depresiva en respuesta a dicho estrés. Sin embargo, por ser la primera vez que se reporta un resultado similar, no hay datos suficientes con los que se puedan contrastar los nuestros para refutar o apoyar la interpretación propuesta. Como suele suceder en relación con las nuevas observaciones, se necesitan más estudios para poder despejar las incógnitas que nacen de nuestras observaciones.

\section{Mecanismos epigenéticos, una hipótesis tentadora}

Hasta aquí se ha descrito una serie de diferencias entre animales con alta y baja inmovilidad que involucran la expresión diferencial de genes y el posible efecto del ambiente como mediador de esa expresión diferencial. Nuestras investigaciones no han profundizado en los posibles mecanismos de regulación que llevan a esa expresión diferencial, sin embargo, los mecanismos de regulación epigenética representan un conjunto de procesos que podrían estar relacionados con algunos de los hallazgos resumidos anteriormente. La regulación epigenética puede definirse como la suma de las alteraciones de la cromatina que colectivamente establecen y propagan diferentes patrones de expresión o silenciamiento a partir de un mismo genoma (Allis, 2006). En otras palabras, representa cambios heredables en la función génica que no pueden explicarse por cambios en la secuencia de ADN (Riggs, Martienssen \& Russo, 1996). La regulación epigenética involucra tres mecanismos complementarios:

i)La metilación o desmetilación del ADN: consiste en la adición o la remoción de grupos metilo de las citocinas en los dinucléotidos citocina-guanina o CpGs.

ii)Las modificaciones químicas en las histonas: involucra la adición o la remoción de grupos químicos en las histonas, que son proteínas fundamentales en los procesos de compactación del genoma y formación de la cromatina. 
iii)La acción de los ARNs no codificantes: corresponden a ARNs que no dirigen la síntesis de proteínas y que participan en el reclutamiento y localización de complejos proteicos en diferentes regiones del genoma.

La acción conjunta de los dos primeros mecanismos permite el reclutamiento de complejos remodeladores lo cual lleva a la formación de estados más compactos o menos compactos de cromatina (i.e., cerrados o abiertos), lo que limita o facilita el acceso de la maquinaria de transcripción, silenciándose o favoreciéndose la expresión de los genes (Vaissière, Sawan \& Herceg, 2008). El tercer mecanismo está íntimamente ligado con los dos primeros pues, en este caso, un ARN no codificante, por ejemplo los ARNs largos no codificantes o los microARNs, al unirse con diferentes proteínas conforman complejos ribonucleoproteicos con capacidad para regular la metilación del ADN y la modificación de histonas lo que, como se mencionó antes, regulan el estado de expresión de los genes (Kaikkonen, Lam \& Glass, 2011).

Una revisión detallada de los mecanismos de control epigenético va más allá de los intereses de este artículo, pero el lector interesado puede acudir a excelentes revisiones sobre el tema (Zhou, Hu \& Lai, 2010; Moore, Le \& Fan, 2013).

Si bien es cierto que las diferencias entre animales con alta y baja inmovilidad en cuanto a la expresión de CRFR1 en el núcleo accumbens podrían estar determinadas por variantes en la secuencia del ADN, también es posible la presencia de "firmas" epigenéticas que determinen mayores o menores niveles de transcripción. Del mismo modo, es posible que mecanismos de regulación epigenética medien la disminución en la expresión de BDNF después de la PNF. De hecho, recientemente se determinó que una sola sesión de la PNF es capaz de inducir modificaciones en histonas (Bilang-Bleuel et al., 2005; Chandramohan, Droste, Arthur \& Reul, 2008), por lo que este tipo de mecanismos de control se perfilan como una posibilidad razonable.

Surge entonces la pregunta lógica: ¿qué eventos pudieron mediar el establecimiento de dichas firmas? Existen varias posibilidades, desde la herencia repetida a lo largo de varias (o muchas) generaciones, hasta eventos durante el desarrollo embrionario y fetal, e inclusive posnatales como por ejemplo el cuido materno (Meaney \& Szyf, 2005). En este sentido, el ambiente juega un papel preponderante en la mediación del establecimiento de tales firmas epigenéticas (revisado en Zhang \& Meaney, 2010). Así, desde la perspectiva de nuestros resultados, el aparente efecto modulador del enriquecimiento ambiental sobre el sistema CRFCRFR1 discutido anteriormente, se perfila como un proceso candidato a ser regulado a través de la metilación del $\mathrm{ADN}$, variaciones en las modificaciones de las histonas y/o la acción de ARNs no codificantes.

Estas posibilidades, por ahora se mantienen en el ámbito de la especulación, pero abren toda una ventana de oportunidades para ampliar los estudios sobre la conducta, sobre los mecanismos neurobiológicos relacionados con ésta y sobre los factores de riesgo para el desarrollo de enfermedades comola depresión. De hecho, se ha propuesto que las modificaciones epigenéticas representan un mecanismo para comprender la relación entre los eventos de vida adversos y los cambios en la expresión de genes que están asociados con la depresión (Dalton, Kolshus \& McLoughlin, 2014). Aunque es un campo de estudio en pleno desarrollo, se perfila como uno de gran importancia para la comprensión de la enfermedad, la identificación de biomarcadores y el desarrollo de nuevas estrategias terapéuticas.

\section{Conclusiones}

En general, la investigación usando modelos animales permite estudiar fenómenos que ocurren en humanos con más profundidad, involucrando diferentes niveles de análisis desde el molecular hasta el conductual. Aunque los datos obtenidos a través de estos modelos no pueden ser trasladados directamente a los humanos, representan una herramienta de gran valor para la realización de estudios y la corroboración de hipótesis cuyo abordaje en seres humanos se vuelve dificultoso y en muchos casos imposible.

Entre las diversas posibilidades, el estudio de las diferencias individuales en la inmovilidad observada en la PNF representa un enfoque útil y novedoso para 
identificar factores relacionados con la depresión. En ese sentido, nuestras investigaciones sugieren que una menor expresión de CRFR1 y una disminución más lenta en los niveles de BDNF, ambos en el núcleo accumbens, son factores relacionados con un mayor riesgo de presentar una conducta relacionada con la depresión. Además, la edad es también un factor importante a tomar en cuenta, pues conforme avanza pueden ocurrir algunos cambios que los protejan contra el surgimiento de este tipo de conductas.

Los mecanismos epigenéticos se posicionan como blancos de interés científico no solamente por su posible participación en el establecimiento de perfiles de expresión génica y el desarrollo de conductas, sino también por su potencial para ser modificados mediante tratamientos farmacológicos o ambientales, como por ejemplo, el enriquecimiento.

\section{Referencias}

Aguilera, G., Nikodemova, M., Wynn, P.C., \& Catt, KJ. (2004). Corticotropin releasing hormone receptors: two decades later. Peptides 25(3), 319-329.

Allis, C.D. (2006). Overview and concepts. In C.D. Allis, T. Jenuwein, \& D. Reinberg (Eds.), Epigenetics (pp.23-62). New York, USA: Cold Spring Harbor Laboratory Press.

Andrade L., Caraveo-Anduaga J.J., Berglund P., Bijl R.V., De Graaf R., Vollebergh W., ...Wittchen HU.(2003). The epidemiology of major depressive episodes: results from the International Consortium of Psychiatric Epidemiology (ICPE) Surveys. International Journal of Methods in Psychiatric Research, 12, 3-21.

Armario, A., \& Nadal, R. (2013). Individual differences and the characterization of animal models of psychopathology: a strong challenge and a good opportunity. Frontiers in Pharmacology, 8, 4:137.

Arrant, A.E., Jemal, H., \& Kuhn, C.M. (2013). Adolescent male rats are less sensitive than adults to the anxiogenic and serotonin-releasing effects of fenfluramine. Neuropharmacology, 65, 213-222.
Bennett, M.R. (2011). The prefrontal-limbic network in depression: Modulation by hypothalamus, basal ganglia and midbrain. Progress in Neurobiology, 93(4), 468-487.

Bilang-Bleuel, A., Ulbricht, S., Chandramohan, Y., De Carli, S., Droste, S.K., \& Reul, J.M. (2005). Psychological stress increases histone H3 phosphorylation in adult dentate gyrus granule neurons: involvement in a glucocorticoid receptordependent behavioural response. European Journal of Neuroscience, 22(7), 1691-1700.

Casey, B.J., Jones, R.M., \&Hare, T.A. (2008). The adolescent brain. Annals of the New York Academy of Sciences, 1124, 111-126.

Chandramohan, Y., Droste, S.K., Arthur, J.S., \& Reul, J.M. (2008). The forced swimming-induced behavioural immobility response involves histone H3 phospho-acetylation and c-Fos induction in dentate gyrus granule neurons via activation of the N-methyl-D-aspartate/extracellular signalregulated kinase/mitogen- and stress-activated kinase signalling pathway. European Journal of Neuroscience, 27(10), 2701-2713.

Consejo Nacional de la Niñez y la Adolescencia. (2009). VII Estado de los derechos de la niñezy la adolescencia en Costa Rica.

Dalton, V.S., Kolshus, E., \& McLoughlin, D.M. (2014). Epigenetics and depression: return of the repressed. Journal of Affective Disorders, 155, 1-12.

Dipietro, J.A. (2012). Maternal stress in pregnancy: considerations for fetal development. Journal of Adolescent Health, 51 (2 Suppl), S3-S8.

Harro, J. (2010). Inter-individual differences in neurobiology as vulnerability factors for affective disorders: implications for psychopharmacology. Pharmacology \& Therapentics, 125(3), 402-422.

Hirschfeld, R.M. (2001). The Comorbidity of Major Depression and Anxiety Disorders: Recognition and Management in Primary Care. The Primary Care Companion to the Journal of Clinical Psychiatry, 3(6), 244-254. 
Jankord, R., Solomon, M.B., Albertz, J., Flak, J.N., Zhang, R., \& Herman, J.P. (2011). Stress vulnerability during adolescent development in rats. Endocrinology, 152(2), 629-638.

Kaikkonen, M.U., Lam, M.T., \& Glass, C.K. (2011). Noncoding RNAs as regulators of gene expression and epigenetics. Cardiovascular Research, 90(3), 430-440.

Kessler, R.C., Berglund, P., Demler, O., Jin, R., Koretz, D., Merikangas, K.R., ... Wang, P.S. (2003). National Comorbidity Survey Replication. The epidemiology of major depressive disorder: results from the National Comorbidity Survey Replication (NCS-R). JAMA, 289, 3095-3105.

Lee, B.H., \& Kim, Y.K. (2010). The roles of BDNF in the pathophysiology of major depression and in antidepressant treatment. Psychiatry Investigation, 7(4), 231-235.

Lépine, J.P., \& Briley, M. (2011). The increasing burden of depression. Journal of Neuropsychiatric Disease and Treatment, 7(Suppl 1), 3-7.

Fattore, L., Piras, G., Corda, M.G., \& Giorgi, O. (2009). The Roman high- and low-avoidance rat lines differ in the acquisition, maintenance, extinction, and reinstatement of intravenous cocaine self-administration. Neuropsychopharmacology, 34, 1091-1101.

Grammatopoulos, D.K., \& Chrousos, G.P. (2002). Functional characteristics of $\mathrm{CRH}$ receptors and potential clinical applications of CRH-receptor antagonists. Trends in Endocrinology \& Metabolism, 13, 436-444.

Lemos, J.C., Wanat, M.J., Smith, J.S., Reyes, B.A., Hollon, N.G., Van Bockstaele, E.J. \& Phillips, P.E. (2012). Severe stress switches CRF action in the nucleus accumbens from appetitive to aversive. Nature, 490 (7420), 402-406.

Mandt, B.H., Allen, R.M., \& Zahniser, N.R. (2009). Individual differences in initial low-dose cocaineinduced locomotor activity and locomotor sensitization in adult outbred female SpragueDawley rats. Pharmacology Biochemistry and Behavior, 91, 511-516.
Meaney, M.J., \& Szyf, M. (2005). Environmental programming of stress responses through DNA methylation: life at the interface between a dynamic environment and a fixed genome. Dialogues in Clinical Neuroscience, 7 (2), 103-123.

Moore, L.D., Le, T., \& Fan, G. (2013). DNA methylation and its basic function. Neuropsychopharmacology, 38 (1), 23-38.

Muschamp, J.W., Van't Veer, A., Parsegian, A., Gallo, M.S., Chen, M., Neve, R.L., Carlezon, W.A. Jr. (2011). Activation of CREB in the nucleus accumbens shell produces anhedonia and resistance to extinction of fear in rats. Journal of Neuroscience, 31, 3095-3103.

Naudon, L., \& Jay, T.M. (2005). Opposite behaviours in the forced swimming test are linked to differences in spatial working memory performances in the rat. Neuroscience, 130 (2), 285-293.

Pawlak, C.R., Ho, Y.J., \& Schwarting, R.K. (2008). Animal models of human psychopathology based on individual differences in novelty-seeking and anxiety. Neuroscience \& Biobehavioral Reviews, 2 (8), 1544-1568.

Peciña, S., Schulkin, J., \& Berridge, K.C. (2006). Nucleus accumbens corticotropin-releasing factor increases cue-triggered motivation for sucrose reward: paradoxical positive incentive effects in stress? BMC Biology, 4, 8.

Porsolt, R.D., Le Pichon, M., \& Jalfre, M. (1977). Depression: a new animal model sensitive to antidepressant treatments. Nature, 266, 730-732.

Riggs, A.D., Martienssen, R.A., \& Russo, V.E.A. (1996). Introduction. In V.E.A. Russo, R.A. Martienssen, A.D. Riggs, \& A.D. Briggs AD (Eds.), Epigenetic mechanisms of gene regulation (pp. 1-4). New York: Cold Spring Harbor Laboratory Press, Cold Spring Harbor.

Rock, P.L., Roiser, J.P., Riedel, W.J., \& Blackwell, A.D. (2013). Cognitive impairment in depression: a systematic review and meta-analysis. Psychological Medicine, 29, 1-12. 
Rupniak, N.M. (2003). Animal models of depression: challenges from a drug development perspective. Behavioural Pharmacology, 14(5-6), 385-390.

Schneider, M. (2013). Adolescence as a vulnerable period to alter rodent behavior. Cell and Tissue Research, 354(1), 99-106.

Schwarting, R.K.W., Thiel, C.M., Muller, C.P., \& Huston, J.P. (1998). Relationship between anxiety and serotonin in the ventral striatum. Neuroreport, 9, 1025-1029.

Sequeira A, \& Fornaguera, J. (2009). Neurobiología de la depresión. Revista Mexicana de Neurociencias, 10(6), 462-478.

Sequeira-Cordero, A., Mora-Gallegos, A., Cuenca-Berger, P., \& Fornaguera-Trías, J. (2013). Individual differences in the immobility behavior in juvenile and adult rats are associated with monoaminergic neurotransmission and with the expression of corticotropin-releasing factor receptor 1 in the nucleus accumbens. Behavioral Brain Research, 252, 77-87.

Sequeira-Cordero, A., Mora-Gallegos, A., CuencaBerger, P., \& Fornaguera-Trías, J. (2014a). Individual differences in the forced swimming test and neurochemical kinetics in the rat brain. Physiology \& Behavior, 128C, 60-69.

Sequeira-Cordero, A., Mora-Gallegos, A., CuencaBerger, P., \& Fornaguera-Trías, J. (2014b). Individual differences in the forced swimming test and the effect of environmental enrichment: Searching for an interaction. Neuroscience, 265C, 95-107.

Shishkina, G.T., Kalinina, T.S., Berezova, I.V., Bulygina, V.V., \& Dygalo, N.N. (2010). Resistance to the development of stress-induced behavioral despair in the forced swim test associated with elevated hippocampal Bcl-xl expression. Behavioral Brain Research, 213 (2), 218-224.

Shumake, J., Barrett, D., \& Gonzalez-Lima, F. (2005). Behavioural characteristics of rats predisposed to learned helplessness: reduced reward sensitivity, increased novelty seeking, and persistent fear memories. Behav Brain Res, 164, 222-230.
Shumake, J., Edwards, E., \& Gonzalez-Lima, F. (2002). Dissociation of septo-hippocampal metabolism in the congenitally helpless rat. Neuroscience, 114, 373-377.

Shumake, J., Poremba, A., Edwards, E., \& GonzalezLima, F. (2000). Congenital helpless rats as a genetic model for cortex metabolism in depression. Neuroreport, 11, 3793-3798.

Simpson, J., \& Kelly, J.P. (2011). The impact of environmental enrichment in laboratory rats: behavioural and neurochemical aspects. Behavioral Brain Research, 222(1), 246-264.

Slattery, D.A., \& Cryan, J.F. (2012). Using the rat forced swim test to assess antidepressant-like activity in rodents. Nature Protocols, 7(6), 1009-1014.

Suganuma, T., \& Workman, J.L. (2011). Signals and combinatorial functions of histone modifications. Anmual Review of Biochemistry, 80, 473-499.

Sun, M.K., \& Alkon, D.L. (2008). Effects of age on susceptibility to the induction of depressive behavior and imipramine in rats. Behavioural Pharmacology, 19(4), 334338.

Taghzout, K., Lamarque, S., Kharouby, M., \& Simon, H. (1999). Interindividual differences in active and passive behaviors in the forced-swimming test implications for animal models of psychopathology. Biological Psycbiatry, 45, 750-758.

Topic, B., Oitzl, M.S., Meijer, O.C., Huston, J.P., \&de Souza Silva, M.A. (2008). Differential susceptibility to extinctioninduced despair and age-dependent alterations in the hypothalamic-pituitary-adrenal axis and neurochemical parameters. Neuropsychobiology, 58 (3-4), 138-153.

Vaissière, T., Sawan, C., \& Herceg, Z. (2008). Epigenetic interplay between histone modifications and DNA methylation in gene silencing. Mutation Research, 659(1-2), 40-48.

Villanueva, R. (2013). Neurobiology of major depressive disorder. Neural Plasticity, 2013, 873278. Doi:org/10.1155/2013/873278

Walsh, R.N., Cummins, R.A. (1976). The Open-Field Test: a critical review. Psychological Bulletin, 83 (3), 482-504. 
Weiss, J.M., Cierpial, M.A., \&West, C.H. (1998). Selective breeding of rats for high and low motor activity in a swim test: toward a new animal model of depression. Pharmacology Biochemistry and Behavior, 61, 49-66.

Yu, H., \& Chen, Z.Y. (2011). The role of BDNF in depression on the basis of its location in the neural circuitry. Acta Pharmacologica Sinica, 32 (1), 3-11.
Zhang, T.Y., \& Meaney, M.J. (2010). Epigenetics and the environmental regulation of the genome and its function. Annual Review of Psychology, 61, 439-466.

Zhou, H., Hu, H., \& Lai, M. (2010). Non-coding RNAs and their epigenetic regulatory mechanisms. Biology of the Cell, 102 (12), 645-655.

Recibido: 2 de abril, 2014

Aceptado: 20 de agosto, 2014 